\title{
Simultaneous Presentation of Thermal and Tactile Sensations Using Multilateral Control under Time Delay
}

\author{
Satoshi Nishimura* Member, \\ Hiroki Kurumatani* \\ Student Member, \\ Yukiko Osawa* \\ Student Member \\ Kazumasa Miura* Member, \\ Yuki Nagatsu* \\ Member \\ Seiichiro Katsura* \\ Senior Member
}

(Manuscript received April 12, 2017, revised Feb. 20, 2018)

\begin{abstract}
This paper presents a method of simultaneously presenting tactile and thermal sensations using multilateral teleoperation. Communication using haptic sensation is expected to be a next-generation communication tool replacing telephone or television. A control technique named multilateral control can transmit haptic sensation to multiple points. In this study, robot manipulators are used for tactile sensation presentation, and Peltier devices are used for rendering thermal sensation. A position encoder is used to measure the position of a robot and also estimate the external force, while temperature and heat flow sensors are used for rendering thermal sensation. A routing method to decouple controllers compensates the effect of a time delay between the systems. Defining a modal transformation matrix in a network system eliminates the interference effect of controllers, which is a well-known problem in bilateral and multilateral teleoperation. The matrix shows how to route the information to realize mode-decoupled control in network systems. To consider the motion range of a robot manipulator, the scaling gain is inserted into the modal transformation matrix. The method also compensates the difference between the response speeds of robot manipulators and Peltier devices. This should be considered because the response speed of a Peltier device is slow compared to that of a robot manipulator. The validity of the proposed method is confirmed through experiments.
\end{abstract}

Keywords: acceleration control, disturbance observer, multilateral control, communication delay, multimodal rendering

\section{Introductions}

There are expectations that communication using haptic sensation to be a practical application. Some of the examples are simple remote communication, remote operation like medical operation or therapy, remote education systems, and so on. A control structure that realizes tactile sensation transmission between two subsystems using an actuator is called bilateral control. In bilateral control, there are two subsystems which are called master and slave systems, and each system tracks the motion of the other system to artificially realize the law of action and reaction so that the impedance of a contacting object can be fed back in front of an operator. The research regarding bilateral control has a long history ${ }^{(1)}$ which started around 1950's ${ }^{(2)}$. For now, there are many control structures like force feedback type bilateral control ${ }^{(3)}$ or impedance feedback type bilateral control ${ }^{(4)}$. Among them, $4 \mathrm{ch}$ bilateral control by acceleration based control is known as a control structure that can transmit precise impedance of a contacting object ${ }^{(5)}$. It can be realized by using the disturbance observer ${ }^{(6)}$ to realize an acceleration based control system. 4ch bilateral control uses modal transformation to decouple each motion mode. The motion of two robots are

\footnotetext{
* Department of System Design Engineering, Keio University 3-14-1, Hiyoshi, Kohoku-ku, Yokohama 223-8522, Japan
}

decomposed to one motion which shows the overall motion as a system and the other motion which shows the relative position between two subsystems, and by decomposing the motions, controllers for each motion can be separately attached.

A control system that transmits tactile sensation not only to two points but to multiple places are called multilateral control $^{(7)}$. In multilateral control, there are three categories, which are multi-master-single-slave type ${ }^{(8)}$, a single-mastermulti-slave type, and multi-master-multi-slave type multilateral control. The structure of the multilateral control is the extended structure of bilateral control. The motion of multirobots are synchronized, and the law of action and reaction can be realized between multiple points. A skill training system ${ }^{(9)}$ or tactile sensation broadcasting system is one of the expected applications of the control. Similar to bilateral control, the motions are decomposed by the quarry matrix ${ }^{(10)}$ so that each motion is decoupled. Research that constructed a multilateral control system for transmitting thermal sensation transmission is also reported ${ }^{(11)}$.

However, it is necessary to consider the effect of time when transmitting tactile sensation to remote places. One report claims that controllers interfere with each other to impede precise sensation transmission when there is a time delay between subsystems ${ }^{(12)}$. Moreover, the stability of the overall control system is deteriorated because the time delay comes inside the control structure. There are some methods 
researched previously. In bilateral teleoperation, $2 \mathrm{ch}$ bilateral teleoperation was proposed ${ }^{(13)}$ whose transparency was optimized. It shows there are two structures (PF and FP) to realize the optimal transparency. To improve the free motion in the PF architecture, a method that uses a high-pass filter in the force control and a damping gain in the slave system $^{(14)}$ was proposed. As for multilateral teleoperation, there is a multilateral control system aiming for stabilizing based on the concept of passivity ${ }^{(15)}$. Force reverse presentation control was proposed in ${ }^{(16)}$, while ${ }^{(17)}$ proposed the control system using wave variable. There are other research which consider the connectivity of subsystems to reduce the operational force of a system ${ }^{(18)}$. Some of the position controllers are removed to attenuate the effect of controller interference. Among them, a control system was proposed which extended a quarry matrix to define motion mode to a system which contains a delay element to decouple controller assigned to each mode $^{(19)}$. By using the method, it is able to construct a multilateral control system with high transparency regardless of time delay.

These research aim for multilateral transmission of tactile sensation; however, control design methodology to simultaneously transmit tactile and thermal sensation using multilateral teleoperation was not established yet. Therefore, in this paper, multilateral control which precisely transmits tactile and thermal sensation to multiple remote places is proposed. Robot manipulators are used as for tactile sensation transmission, while Peltier devices are used for the use of thermal sensation transmission. By using the disturbance observer, motors and Peltier devices can be treated in the same manner. A control structure which decouples each mode is constructed into both tactile and thermal sensation transmission system. To consider the motion range of a robot manipulator, the scaling gain is inserted into the modal transformation matrix. The response speed of Peltier devices is slow compared to that of motors. Therefore, to correct the timing of presenting sensations, information regarding tactile information is artificially buffered. In this paper, the number of subsystems for communication is three, and the delay time between subsystems are $50 \mathrm{~ms}$ plus $5 \mathrm{~ms}$ jitter. Under this condition, the paper realizes a precise sensation transmission system.

\section{Acceleration and Heat Flow Control Using Disturbance Observer}

In this chapter, an acceleration based control system and a heat flow control system are constructed to a motor and Peltier device by using the Disturbance Observer.

\subsection{Disturbance Observer for Motor System}

A robot manipulator is used as an actuator for transmitting tactile sensation. Driving force $F$ is generated by applying current $I$ to a motor:

$$
F=K_{t} I, \cdots \cdots \cdots \cdots \cdots \cdots \cdots \cdots \cdots \cdots \cdots \cdots \cdots \cdots \cdots \cdots \cdots \cdots \cdots
$$

where $K_{t}$ stands for the torque constant.

Motors, as a mechanical system, follows the motion equation as

$$
M \frac{d^{2} X}{d t^{2}}=F^{\mathrm{ref}}-F^{\mathrm{dis}}, \ldots \ldots \ldots \ldots \ldots \ldots \ldots \ldots \ldots \ldots
$$

where $M$ stands for the mass of a motor. The block diagram

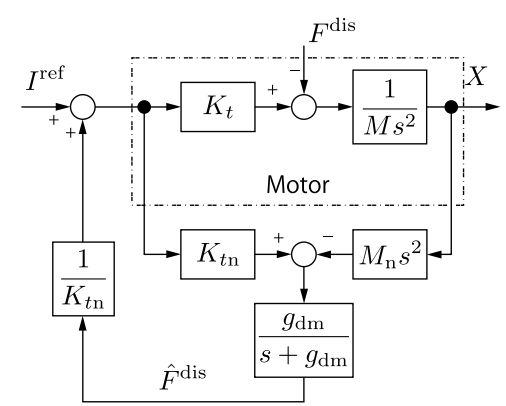

Fig. 1. Motor with disturbance observer

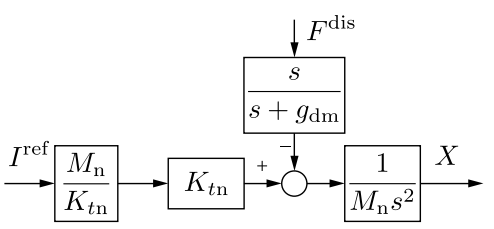

Fig. 2. Acceleration control of a motor system

when the disturbance observer is applied to a motor whose model is based on (2) is shown in Fig. 1. By applying the disturbance observer, a control system which is robust against disturbances such as gravity or friction inside a motor can be constructed. The equivalent block diagram of Fig. 1 is expressed as Fig. 2. Some of the parameters can be nominalized and fixed by using the disturbance observer.

2.2 Disturbance Observer for Peltier Device This paper uses the Peltier device as an actuator for presenting thermal sensation. The disturbance observer is also used to the Peltier device to construct the heat flow control system.

The Peltier device is actuated by applying DC current to generate heat transfer as

$$
q=-\alpha T I+\frac{1}{2} R_{\mathrm{p}} I^{2},
$$

where $I, \alpha, T, R_{\mathrm{p}}$ stand for applied current, Seebeck coefficient, the temperature of the Peltier device, and the electrical resistance inside the Peltier device, respectively. The Peltier device absorbs heat when a forward current is applied; therefore, there is a minus sign in (3). The second term in (3) represents the Joule heat.

The modeling of the Peltier device is done using the thermal network method ${ }^{(20)}$. By using the method, the temperature difference of the Peltier device can be expressed as

$$
C_{\mathrm{p}} \frac{d T}{d t}=-q^{\mathrm{ref}}+q^{\mathrm{dis}}
$$

where $C_{\mathrm{p}}, q_{\mathrm{in}}$, and $q_{\mathrm{dis}}$ stand for the heat conductance, heat flow reference, and the disturbance heat flow of the Peltier device, respectively. The block diagram when the disturbance observer is applied to the Peltier device is shown in Fig. 3, and the equivalent transformation is shown in Fig. 4. By using the disturbance observer, the control structure which is robust against modeling errors, Joule heat, and disturbance heat flow is constructed.

The block diagrams of motors and Peltier devices can be treated as Figs. 2 and 4 by applying the disturbance observers. 


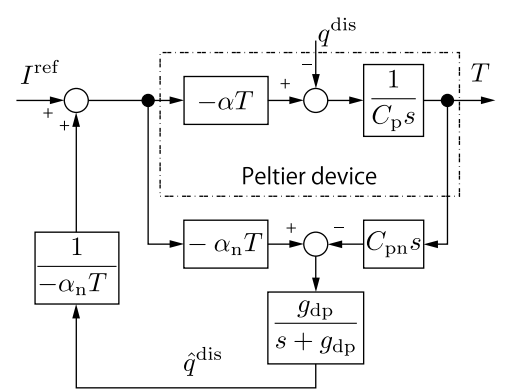

Fig. 3. Peltier device with disturbance observer

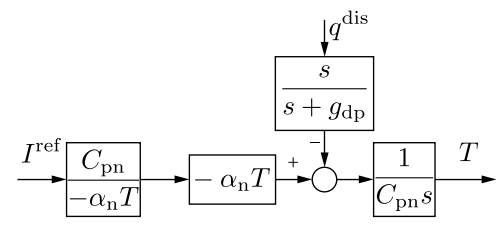

Fig. 4. Acceleration control of a thermal system

\section{Modal Space Decoupled Multilateral Control under Time Delay}

The section discusses the decoupling control of the multilateral control system under time delay.

3.1 Control Goals The control goals of multilateral control when the number of subsystems is three are shown as

$$
\left\{\begin{array}{r}
F_{1}+F_{2}+F_{3}=0 \\
-X_{1}+2 X_{2}-X_{3}=0 \\
-X_{1}-X_{2}+2 X_{3}=0
\end{array}\right.
$$

where the subscript $i$ stands for the response of subsystem $i$. On the other hand, the control goals for transmitting thermal sensation is given as

$$
\left\{\begin{array}{r}
q_{1}+q_{2}+q_{3}=0 \\
-T_{1}+2 T_{2}-T_{3}=0 \\
-T_{1}-T_{2}+2 T_{3}=0
\end{array}\right.
$$

In order to realize each control goal, a quarry matrix is used to decompose the system into each mode ${ }^{(10)}$. The quarry matrix is represented as

$$
Q=\left[\begin{array}{rrr}
1 & 1 & 1 \\
-1 & 2 & -1 \\
-1 & -1 & 2
\end{array}\right]
$$

The matrix transforms the motion of each subsystem into one motion that represents the overall motion of the system and two motions that describes the relationship between each subsystem. Controllers are placed for each motion to control each motion individually.

\subsection{Conventional 4ch Multilateral Teleoperation}

The acceleration reference of $i$ th subsystem using 4ch multilateral control under a time delay is given as

$$
s^{2} X_{i}^{\mathrm{ref}}=-C_{\mathrm{c}} \boldsymbol{S}_{\mathrm{c}} \boldsymbol{Q}_{i} \boldsymbol{F}-C_{\mathrm{d}} \boldsymbol{S}_{\mathrm{d}} \boldsymbol{Q}_{i} \boldsymbol{X},
$$

where $C_{\mathrm{c}}$ and $C_{\mathrm{d}}$ stand for the controllers for the common and differential modes, respectively. $\boldsymbol{X}$ and $\boldsymbol{F}$ are the state matrices which is $3 \times 1$ matrix, and $\boldsymbol{S}_{\mathrm{c}}=\left[\begin{array}{lll}1 & 0 & 0\end{array}\right]^{T}$ and $S_{\mathrm{d}}=\left[\begin{array}{lll}0 & 1 & 1\end{array}\right]^{T}$, which are called the selection matrices. The modal transformation matrix in each subsystem including the

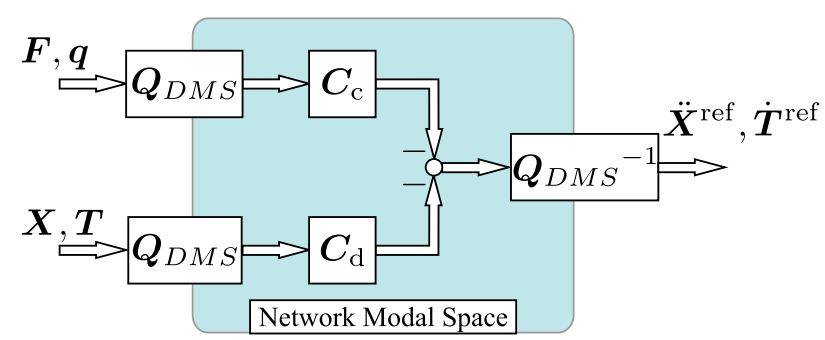

Fig. 5. Modal transformation taking network delay into account

delay time as an element of the matrix is expressed as

$$
\begin{aligned}
& \boldsymbol{Q}_{1}=\left[\begin{array}{ccc}
1 & e^{-T_{12} s} & e^{-T_{13} s} \\
1 & -e^{-T_{12} s} & 0 \\
1 & 0 & -e^{-T_{13} s}
\end{array}\right], \\
& \boldsymbol{Q}_{2}=\left[\begin{array}{ccc}
e^{-T_{21} s} & 1 & e^{-T_{23} s} \\
-e^{-T_{21} s} & 1 & 0 \\
0 & 1 & -e^{-T_{23} s}
\end{array}\right], \\
& \boldsymbol{Q}_{3}=\left[\begin{array}{ccc}
e^{-T_{31} s} & e^{-T_{32} s} & 1 \\
-e^{-T_{31} s} & 0 & 1 \\
0 & -e^{-T_{32} s} & 1
\end{array}\right],
\end{aligned}
$$

where $T_{i j}$ stands for the delay time from subsystem $j$ to $i$.

Because there is a time delay in networks when transmitting information to remote places, the aforementioned control goals cannot be realized. There are three modes that can be defined based on the received information, and the number of a subsystem is three; therefore, total number of the modes which can be defined is 9. Actually, the number of mode controllable is the same as the number of a subsystem. When the matrix is different between each other, the inverse of the modal transformation matrix cannot be defined, which is the biggest problem in case of a time delay.

In this paper, control goals are modified considering the delay time between subsystems. A multi-master-single-slave system is assumed in this paper; therefore, a control structure, which is able to feedback the impedance of an object at a slave system to master systems, is constructed.

3.3 Decoupling of Motion Mode The concept of the modal transformation matrix is shown in Fig. 5. By regarding the delay element as a coefficient which is attached during the modal transformation, it becomes possible to determine the motion mode that integrates the effect of a time delay between subsystems.

In this paper, subsystem 1 is the slave system, and subsystem 2 and 3 are master systems, and in order to compensate the limitation of motion area, the motion of master systems are scaled by using a scaling gain. The mode decoupling matrix $\boldsymbol{Q}_{\mathrm{DMS}}$ that is used in this paper regarding tactile sensation is defined as

$$
\boldsymbol{Q}_{\mathrm{DMS}}^{\mathrm{f}}=\left[\begin{array}{ccc}
1 & a e^{-T_{12} s} & b e^{-T_{13} s} \\
-e^{-T_{21} s} & 2 a & -b e^{-T_{23} s} \\
-e^{-T_{31} s} & -a e^{-T_{32} s} & 2 b
\end{array}\right], \cdots \cdots
$$

where $a$ and $b$ stand for the scaling gain of subsystem 2 and 3, respectively. The matrix for thermal sensation transmission is defined as 


$$
\begin{aligned}
& \operatorname{det}\left(\boldsymbol{Q}_{\mathrm{DMS}}^{\mathrm{t}}\right)=a b\left(4-e^{-\left(T_{23}+T_{32}\right) s}+2 e^{-\left(T_{12}+T_{21}\right) s}+2 e^{-\left(T_{13}+T_{31}\right) s}+e^{-\left(T_{12}+T_{23}+T_{31}\right) s}+e^{-\left(T_{13}+T_{21}+T_{32}\right) s}\right) \cdots \cdots \cdots \cdots \cdots \cdots \cdots \\
& \boldsymbol{Q}_{\mathrm{DMS}}^{\mathrm{t}}{ }^{-1}=\left[\begin{array}{rrr}
-a b\left(e^{-\left(T_{23}+T_{32}\right) s}-4\right) & -a b\left(2 e^{-T_{12} s}+e^{-\left(T_{13}+T_{32}\right) s}\right) & -a b\left(2 e^{-T_{13} s}+e^{-\left(T_{12}+T_{23}\right) s}\right) \\
b\left(2 e^{-T_{21} s}+e^{-\left(T_{23}+T_{31}\right) s}\right) & b\left(2+e^{-\left(T_{13}+T_{31}\right) s}\right) & b\left(e^{-T_{23} s}-e^{-\left(T_{13}+T_{21}\right) s}\right) \\
a\left(2 e^{-T_{31} s}+e^{-\left(T_{21}+T_{32}\right) s}\right) & a\left(e^{-T_{32} s}-e^{-\left(T_{12}+T_{31}\right) s}\right) & a\left(2+e^{-\left(T_{12}+T_{21}\right) s}\right)
\end{array}\right] \cdots \cdots \cdots \cdots \cdots \cdots \cdots \cdots \cdots \cdots
\end{aligned}
$$

$$
\boldsymbol{Q}_{\mathrm{DMS}}^{\mathrm{t}}=\left[\begin{array}{ccc}
1 & e^{-T_{12} s} & e^{-T_{13} s} \\
-e^{-T_{21} s} & 2 & -e^{-T_{23} s} \\
-e^{-T_{31} s} & -e^{-T_{32} s} & 2
\end{array}\right] \ldots \ldots \ldots
$$

The control goals are modified from (5) and (6) into

$$
\begin{aligned}
& \left\{\begin{array}{rl}
F_{1}+a e^{-T_{12} s} F_{2}+b e^{-T_{13} s} F_{3} & =0 \\
-e^{-T_{21} s} X_{1}+2 a X_{2}-b e^{-T_{23} s} X_{3} & =0 \\
-e^{-T_{31} s} X_{1}-a e^{-T_{32} s} X_{2}+2 b X_{3} & =0
\end{array}, \cdots(1)\right.
\end{aligned}
$$

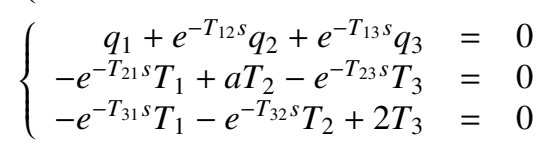

By defining the modal transformation matrix as (12), it is possible to define a modal transformation that includes network space $^{(19)}$.

By calculating the inverse matrices of (12) and (13), the inverse mapping matrices corresponding to (12) and (13) can be derived. In case of the perfect implementation, it is required to calculate the inverse of the determinant which is shown in (16) that is placed at the top of this page. It is difficult to implement the determinant; therefore, in this paper, the determinant is implemented as 1 . The effect of the determinant appears in the modal space, which becomes a coefficient of a controller inside the modal space. The gains of each mode are determined by plotting the Nyquist plot. The inverse matrix used in this paper is shown in (17) that is also placed at the top of this page.

It is able to know how the information is routed by looking at the element of the inverse matrix. Compared to the conventional method, the stability margin is limited because the proposed method contains a large number of time delay. However, the proposed method is able to transmit precise sensation to remote places within the stability is assured because the controllers do not interfere with each other.

Since the proposal is based on the modal transportation technique, it is able to control each motion separately. It means it is able to eliminate the interference of controllers which is one of the problems in conventional multilateral teleoperation.

3.4 Simultaneous Presentation of Haptic Sensation

Since response speed of a Peltier device is slower than a robot manipulator, the quarry matrix for force sensation transmission artificially inserts a buffer time to correct the timing for the simultaneous presentation. The proposed quarry matrix is expressed as

$$
\boldsymbol{Q}_{\mathrm{DMS}}^{\mathrm{f}}=\left[\begin{array}{ccc}
1 & a e^{-\left(T_{12}+T_{\mathrm{b}}\right) s} & b e^{-\left(T_{13}+T_{\mathrm{b}}\right) s} \\
-e^{-\left(T_{21}+T_{\mathrm{b}}\right) s} & 2 a & -b e^{-\left(T_{23}+T_{\mathrm{b}}\right) s} \\
-e^{-\left(T_{31}+T_{\mathrm{b}}\right) s} & -a e^{-\left(T_{32}+T_{\mathrm{b}}\right) s} & 2 b
\end{array}\right],
$$

where $T_{\mathrm{b}}$ stands for the buffered time. The timing that human recognizes the hardness and hotness of a contact object can be corrected by additionally inserting a delay element.

The method has a high affinity to network systems because it naturally includes a time delay element inside the control system. The buffer can also be used for jitter buffering.

3.5 Stability Analysis in the Modal Space The following subsection analyzes the stability of the proposed method in the modal space. The motor system is used as an example. The acceleration reference for each subsystem is given as

$$
\begin{aligned}
s^{2} \boldsymbol{X}^{\mathrm{ref}}= & -C_{\mathrm{c}}^{\mathrm{f}} \frac{\operatorname{det}\left(\boldsymbol{Q}_{\mathrm{DMS}}^{\mathrm{f}}\right)}{9 a b} \boldsymbol{Q}_{\mathrm{DMS}}^{\mathrm{f}}{ }^{-1} \boldsymbol{S}_{\mathrm{c}} \boldsymbol{Q}_{\mathrm{DMS}}^{\mathrm{f}} \boldsymbol{F} \\
& -C_{\mathrm{d}}^{\mathrm{f}} \frac{\operatorname{det}\left(\boldsymbol{Q}_{\mathrm{DMS}}^{\mathrm{f}}\right)}{9 a b} \boldsymbol{Q}_{\mathrm{DMS}}^{\mathrm{f}}{ }^{-1} \boldsymbol{S}_{\mathrm{d}} \boldsymbol{Q}_{\mathrm{DMS}}^{\mathrm{f}} \boldsymbol{X}, \cdots
\end{aligned}
$$

where $\boldsymbol{S}_{\mathrm{c}}=\operatorname{diag}\left[\begin{array}{lll}1 & 0 & 0\end{array}\right]$ and $\boldsymbol{S}_{\mathrm{d}}=\operatorname{diag}\left[\begin{array}{lll}0 & 1 & 1\end{array}\right]$. The acceleration reference for the modal space is calculated as

$$
s^{2} \boldsymbol{X}_{m}^{\mathrm{ref}}=-C_{\mathrm{c}}^{\mathrm{f}} \frac{\operatorname{det}\left(\boldsymbol{Q}_{\mathrm{DMS}}^{\mathrm{f}}\right)}{9 a b} \boldsymbol{S}_{\mathrm{c}} \boldsymbol{F}_{m}-C_{\mathrm{d}}^{\mathrm{f}} \frac{\operatorname{det}\left(\boldsymbol{Q}_{\mathrm{DMS}}^{\mathrm{f}}\right)}{9 a b} \boldsymbol{S}_{\mathrm{d}} \boldsymbol{X}_{m},
$$

where the subscript $m$ stands for the variable of the modal space. An environmental impedance matrix and its modal space expression are given as follows

$$
\begin{aligned}
& \boldsymbol{Z}=\operatorname{diag}\left\{Z_{1}, Z_{2}, Z_{3}\right\}, \ldots \ldots \ldots \ldots \ldots \ldots \ldots \\
& \boldsymbol{Z}_{m}=\boldsymbol{Q}_{\mathrm{DMS}}^{\mathrm{f}} \boldsymbol{Z} \boldsymbol{Q}_{\mathrm{DMS}}^{\mathrm{f}}{ }^{-1}
\end{aligned}
$$

Then, the block diagrams of the modal spaces are obtained as Figs. 6 and 7.

The open loop transfer functions for each mode are calculated as

$$
\begin{aligned}
& G_{\mathrm{d}}^{\mathrm{open}}=\frac{\operatorname{det}\left(\boldsymbol{Q}_{\mathrm{DMS}}^{\mathrm{f}}\right)}{9 a b} C_{\mathrm{d}}^{\mathrm{f}} \frac{1}{s^{2}}, \\
& G_{\mathrm{c}}^{\mathrm{open}}=\frac{1}{9} C_{\mathrm{c}}^{\mathrm{f}} \tilde{Z}_{11}^{\mathrm{f}} \frac{1}{s^{2}}, \cdots \cdots \\
& \tilde{Z}_{11}^{\mathrm{f}}=\frac{\operatorname{det}\left(\boldsymbol{Q}_{\mathrm{DMS}}^{\mathrm{f}}\right)}{a b} Z_{11}, \cdots
\end{aligned}
$$

The controller gains are determined by using (23) and (24). The differential mode is assumed to be well controlled so that the disturbance to the common mode is nearly zero. In the following, all of the delay time including the artificial buffer is assumed as $50 \mathrm{~ms}$.

At first, the Nyquist plot of the differential mode is obtained as shown in Fig. 8. The controller is $C_{\mathrm{d}}^{\mathrm{f}}=K_{\mathrm{p}}+2 \sqrt{K_{\mathrm{p}}} s$. The determinant of the proposed quarry matrix determines the upper limitation of the gains of the controller. As it is 


$$
\boldsymbol{H}^{\mathrm{f}}=\left[\begin{array}{ccc}
\frac{C_{\mathrm{f}} s^{2}}{e^{-\left(T_{31}+T_{\mathrm{b}}\right) s}} & -a \frac{e^{-\left(T_{32}+T_{\mathrm{b}}\right) s}}{e^{-\left(T_{31}+T_{\mathrm{b}}\right) s}} & -b \frac{1}{e^{-\left(T_{31}+T_{\mathrm{b}}\right) s}} \\
\frac{e^{-\left(T_{21}+T_{13}+2 T_{\mathrm{b}}\right) s}+2 e^{-\left(T_{23}+T_{\mathrm{b}}\right) s}}{e^{-\left(T_{12}+T_{23}+2 T_{\mathrm{b}}\right) s}+2 e^{-\left(T_{13}+T_{\mathrm{b}}\right) s}} & 0 & 0 \\
\frac{4-e^{-\left(T_{12}+T_{21}+2 T_{\mathrm{b}}\right) s}}{e^{-\left(T_{12}+T_{23}+2 T_{\mathrm{b}}\right) s}+2 e^{-\left(T_{13}+T_{\mathrm{b}}\right) s}} & 0 & 0
\end{array}\right]
$$

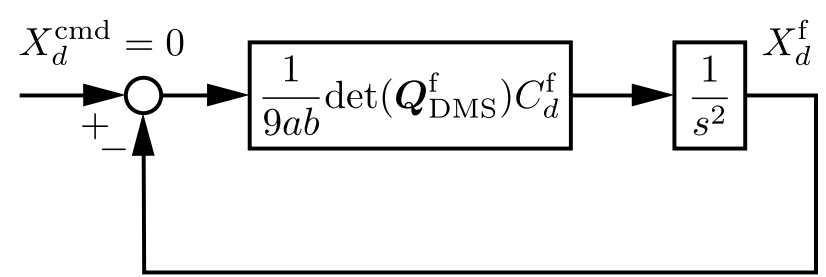

Fig. 6. Block diagram of the differential mode for the motor system

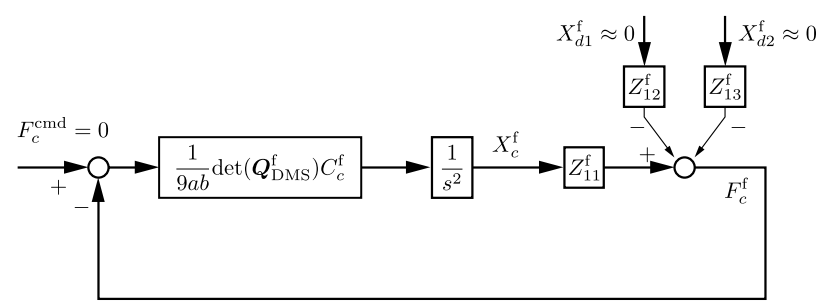

Fig. 7. Block diagram of the common mode for the motor system

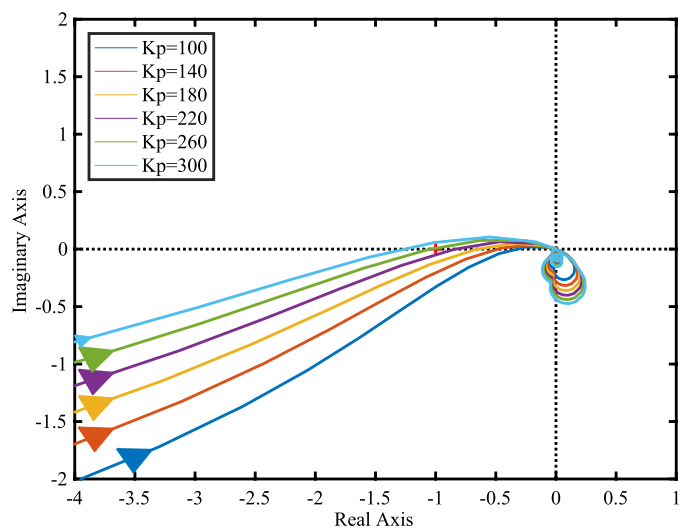

Fig. 8. Nyquist plot of the differential mode

shown in Fig. 8, the differential mode becomes unstable as the $\mathrm{P}$ gain becomes large. It also indicates that the more buffer it is inserted, the more narrow the stability margin becomes.

The Nyquist plot of the common mode is shown in Figs. 9 and 10. It shows how stability varies when the impedance of the environment changes. The impedance of an operator in subsystem 2 is assumed as $Z_{2}=100+100 s$, and the common mode controller is assumed as $C_{\mathrm{c}}^{\mathrm{f}}=1.0$. Figure 9 shows the situation when the environment is soft, and the situation with a hard object is assumed in Fig. 10. Figure 9 shows the proposed system is unstable when the environment is very soft. However, Fig. 10 shows the common mode is stable when the object is hard. Therefore, the analysis gives that the proposed

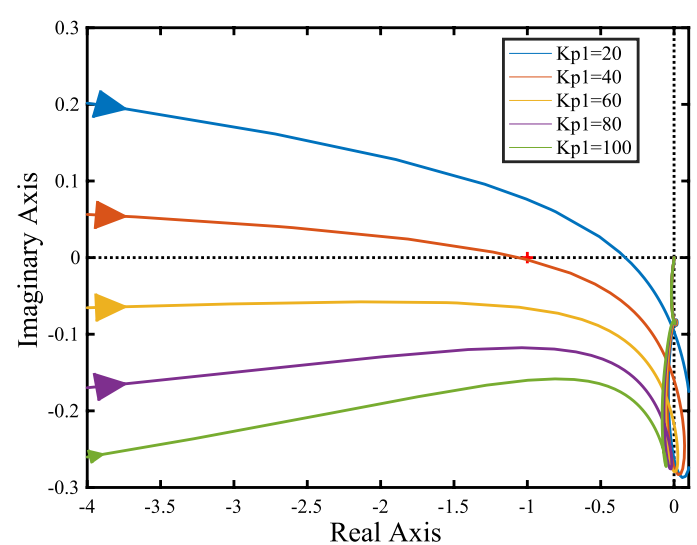

Fig. 9. Nyquist plot for the common mode when the environment is soft $\left(Z_{1}=K_{\mathrm{p} 1}+10 s\right)$

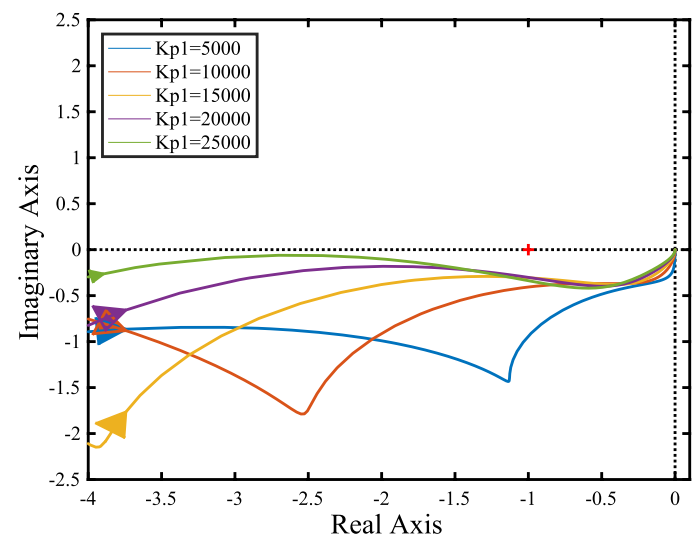

Fig. 10. Nyquist plot for the common mode when the environment is soft $\left(Z_{1}=K_{\mathrm{p} 1}+100 s\right)$

method is suitable for the hard contact motion.

3.6 Fundamental Matrix for Performance Evaluation

The performance of the proposed method can be analytically evaluated by using a fundamental matrix. The matrix shows the relationship between variables and it is defined as

$$
\left[\begin{array}{lll}
F_{1} & X_{2} & X_{3}
\end{array}\right]^{T}=\boldsymbol{H}\left[\begin{array}{lll}
X_{1} & F_{2} & F_{3}
\end{array}\right]^{T} .
$$

The fundamental matrix for tactile sensation transmission system is calculated as shown in (27). The proposed method is free from the interference because $h_{22}, h_{23}, h_{32}$, and $h_{33}$, which show the interference term, is 0 . The elements being 0 indicate that the proposed method can realize the desired control goals that are expressed in (14).

The matrix for thermal sensation transmission system is obtained as 


$$
\boldsymbol{H}^{\mathrm{t}}=\left[\begin{array}{ccc}
\frac{C_{\mathrm{f}} s^{2}}{e^{-T_{31} s}} & -\frac{e^{-T_{32} s}}{e^{-T_{31} s}} & -\frac{1}{e^{-T_{31} s}} \\
\frac{e^{-\left(T_{21}+T_{13}\right) s}+2 e^{-T_{23} s}}{e^{-\left(T_{12}+T_{23}\right) s}+2 e^{-T_{13} s}} & 0 & 0 \\
\frac{4-e^{-\left(T_{12}+T_{21}\right) s}}{e^{-\left(T_{12}+T_{23}\right) s}+2 e^{-T_{13} s}} & 0 & 0
\end{array}\right] .
$$

The control goals of the thermal sensation transmission shown in (15) are also realized in the proposed method even though there are time delays between every subsystem. It is obvious that both sensation transmission systems have very high transparency.

3.7 Operationality and Reproducibility The following shows the performance of the proposal by using the indices operationality and reproducibility. For the simplicity of the time delay, upstream and downstream delay times are assumed to be the same as

$$
T_{12}=T_{21}=\tau_{1}, T_{23}=T_{32}=\tau_{2}, T_{31}=T_{13}=\tau_{3} .
$$

The indices can be obtained by using the fundamental matrix; however, for the symmetry of the matrix, the indices are calculated by using the following matrix:

$$
\left[\begin{array}{lll}
X_{1} & X_{2} & X_{3}
\end{array}\right]^{T}=\frac{1}{P_{\text {den }}} \boldsymbol{P}_{\text {num }}\left[\begin{array}{lll}
F_{1} & F_{2} & F_{3}
\end{array}\right]^{T} .
$$

By calculating the transfer functions from the position response of a master system to the force response of the master system, the indices are obtained as

$$
\begin{aligned}
F_{2} & =\frac{P_{\text {den }}\left(P_{\mathrm{den}}-P_{11} Z_{1}\right)}{P_{22} P_{\mathrm{den}}+\left(P_{12} P_{21}-P_{11} P_{12}\right) Z_{1}} X_{2} \\
& =\left(P_{r 2} Z_{1}+P_{o 2}\right) X_{2}, \ldots \ldots \ldots \ldots \ldots \ldots \\
F_{3} & =\frac{P_{\mathrm{den}}\left(P_{\mathrm{den}}-P_{11} Z_{1}\right)}{P_{33} P_{\mathrm{den}}+\left(P_{13} P_{31}-P_{11} P_{33}\right) Z_{1}} F_{3} \\
& =\left(P_{r 3} Z_{1}+P_{o 3}\right) X_{3} . \ldots \ldots \ldots \ldots \ldots \ldots
\end{aligned}
$$

The elements for the tactile sensation presentation are calculated as

$$
\begin{aligned}
& P_{11}^{\mathrm{f}}=a b\left(4-e^{-2\left(\tau_{2}+T_{\mathrm{b}}\right) s}\right), \\
& P_{21}^{\mathrm{f}}=b\left(2 e^{-\left(\tau_{1}+T_{\mathrm{b}}\right) s}+e^{-\left(\tau_{2}+\tau_{3}+2 T_{\mathrm{b}}\right) s}\right), \\
& P_{31}^{\mathrm{f}}=a\left(2 e^{-\left(\tau_{3}+T_{\mathrm{b}}\right) s}+e^{-\left(\tau_{1}+\tau_{2}+2 T_{\mathrm{b}}\right) s}\right), \\
& P^{\mathrm{f}}=\frac{K_{\mathrm{hp}}}{9 a b s^{2}}\left[\begin{array}{ccc}
P_{11}^{\mathrm{f}} & a e^{-\left(\tau_{1}+T_{\mathrm{b}}\right) s} P_{11}^{\mathrm{f}} & b e^{-\left(\tau_{3}+T_{\mathrm{b}}\right) s} P_{11}^{\mathrm{f}} \\
P_{21}^{\mathrm{f}} & a e^{-\left(\tau_{1}+T_{\mathrm{b}}\right) s} P_{21}^{\mathrm{f}} & b e^{-\left(\tau_{3}+T_{\mathrm{b}}\right) s} P_{21}^{\mathrm{f}} \\
P_{31}^{\mathrm{f}} & a e^{-\left(\tau_{1}+T_{\mathrm{b}}\right) s} P_{31}^{\mathrm{f}} & b e^{-\left(\tau_{3}+T_{\mathrm{b}}\right) s} P_{31}^{\mathrm{f}}
\end{array}\right] .
\end{aligned}
$$

Setting $a=b=1$ and $T_{\mathrm{b}}=0$, it becomes the elements for the thermal sensation presentation.

When assuming all of the delay time as the same, the operationality and reproducibility of subsystems 2 and 3 become the same. The indices of subsystem 2 in the proposal are calculated as

$$
P_{r 2}=\frac{4-e^{-2\left(\tau_{2}+T_{\mathrm{b}}\right) s}}{e^{-\left(\tau_{1}+T_{\mathrm{b}}\right) s}\left(2 e^{-\left(\tau_{1}+T_{\mathrm{b}}\right) s}+e^{-\left(\tau_{2}+\tau_{3}+2 T_{\mathrm{b}}\right) s}\right) C_{\mathrm{c}}},
$$

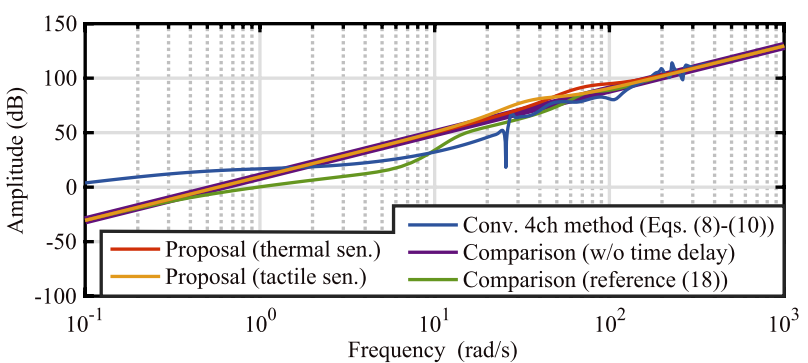

Fig. 11. Operationality when the environment is stiff

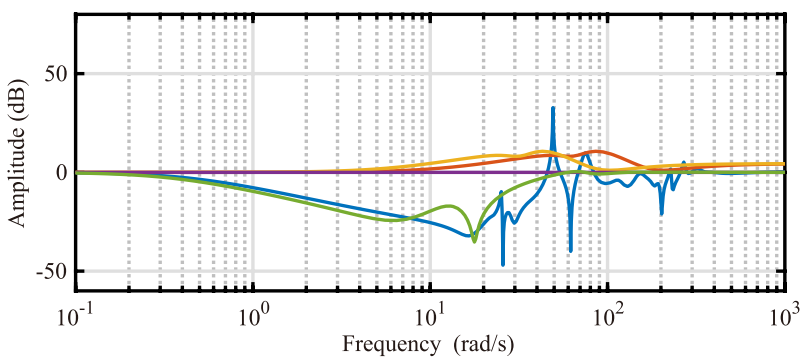

Fig. 12. Reproducibility when the environment is stiff

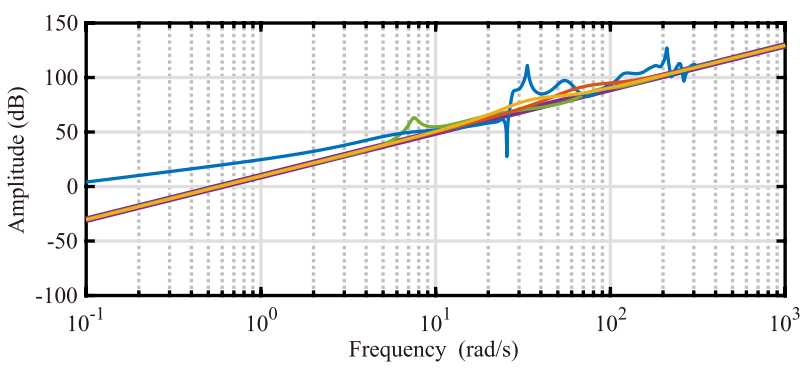

Fig. 13. Operationality when the environment is soft

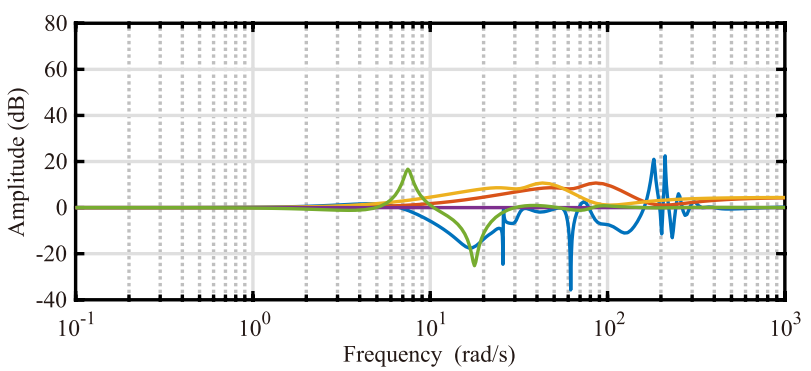

Fig. 14. Reproducibility when the environment is soft

$$
P_{o 2}=\frac{9 s^{2}}{e^{-\left(\tau_{1}+T_{\mathrm{b}}\right) s}\left(2 e^{-\left(\tau_{1}+T_{\mathrm{b}}\right) s}+e^{-\left(\tau_{2}+\tau_{3}+2 T_{\mathrm{b}}\right) s}\right) C_{\mathrm{c}}} .
$$

It shows that the scaling gains do not affect the performance of both the operationality and reproducibility while the artificial buffer affects both indices. It clearly shows the controller for the differential mode does not appear in the indices.

The performance is compared in the following. There are several methods compared in this analysis: the multilateral teleoperation using (9), (10), and (11), multilateral control without time delay, and the system of ${ }^{(18)}$. The parameters for the Bode plot are the same as those in the experiments. The cut-off frequency of the communication disturbance observer used in ${ }^{(18)}$ is set as $100 \mathrm{rad} / \mathrm{s}$. Figures 11 to 14 show the Bode diagram of the operationality and the reproducibility. 
Figures 11 and 12 are the situation when the environment is hard, and Figs. 13 and 14 are the case with a soft object. The red line indicates the index for the thermal sensation presentation, while the yellow line shows that of tactile sensation transmission. The lines of the proposal do not change even the impedance changes. This is because the impedance in the denominator of (30) and (31) disappears in the proposed method. In the hard contact, it can be said that the proposed methods have the better performance than the conventional methods even though the amplitude of the index is over $0 \mathrm{~dB}$ around $40 \mathrm{rad} / \mathrm{s}$. As for the operationality, the proposal has the double integrator-like characteristics regardless of the time delay. The analysis shows the proposal is effective in hard contact motion.

The largest difference between the proposal and the conventional $4 \mathrm{ch}$ multilateral teleoperation is the operationality in the low frequency area, where human operates the system. Moreover, even though the reproducibility of the proposal becomes larger than $0 \mathrm{~dB}$ around 10 to $100 \mathrm{rad} / \mathrm{s}$, the index shows the proposal is superior to the conventional method in low frequency when the environment is stiff.

Since the reference values for the differential mode are generated at the master systems, the structure is similar to that of the $2 \mathrm{ch}$ transparency optimized controller, which is called a PF structure ${ }^{(13)}$. However, one of the differences between the proposal and the $2 \mathrm{ch}$ controller is that the position controllers of the proposed method do not apparently appear in the transparency, which means the position controllers work as an internal force. In a 2 ch controller, it is required to design both force and position controllers to considering the stability of the contact motion.

\section{Experiments}

In order to confirm the validity of the proposed method, experiments were conducted. The experimental setup is shown in Fig. 15. Assuming the sensation transmission to a remote place, delay time was $50 \mathrm{~ms}$ constant delay with $5 \mathrm{~ms}$ jitter in the experiments. 6 DOF robot manipulators were placed as an actuator for transmitting force sensation, and the Peltier devices are placed at the end effector of the manipulators for thermal sensation transmission. One axis is used for the multilateral teleoperation, and position control is used in another axis. The coordinate transformation for master 2 and slave is based on the study of ${ }^{(21)}$. For the simplicity of the coordinate transformation for master 1 , a 6 th order quarry matrix is used. A temperature and heat flow sensors are attached to the Peltier device, and a position encoder is used to detect the position of each motor. As for the force response, a reaction force observer is used to estimate the external force.

The parameters used in the experiments are shown in Table 1. A cold aluminum plate was used as a contact object. After the contact motion becomes steady, push and pull motion to the environment was conducted to examine whether each controller is decoupled from the other controllers.

The method which does not decouple the modes was selected as a conventional method. The modal transformation matrices in the conventional method are expressed in (9), (10), and (11). As mentioned in the previous sections, it is usual 4ch multilateral teleoperation. The experimental results of the conventional method are shown in Figs. 16 and 17.

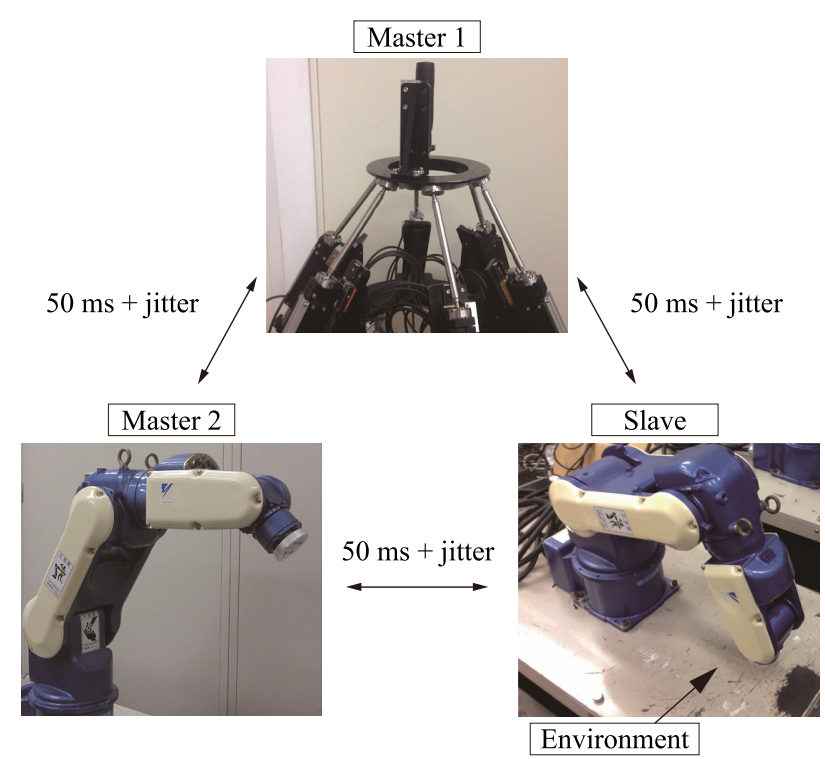

Fig. 15. Experimental setup

Table 1. Experimental parameters

\begin{tabular}{|c|l|c|}
\hline Parameter & Description & Value \\
\hline \hline$K_{\mathrm{pm}}$ & P gain in position control & 160.0 \\
$K_{\mathrm{dm}}$ & D gain in position control & 25.3 \\
$K_{\mathrm{fm}}$ & P gain in force control & 1.0 \\
$g_{\mathrm{dm}}$ & CoF of DOB for a motor & $1000.0 \mathrm{rad} / \mathrm{s}$ \\
$g_{\mathrm{rm}}$ & CoF of RFOB for a motor & $1000.0 \mathrm{rad} / \mathrm{s}$ \\
$K_{\mathrm{tp}}$ & P gain in temperature control & 0.7 \\
$K_{\mathrm{hp}}$ & P gain in heat flow control & 1.0 \\
$g_{\mathrm{dp}}$ & CoF of DOB for a Peltier device & $1.0 \mathrm{rad} / \mathrm{s}$ \\
$g_{\mathrm{rp}}$ & CoF of RFOB for a Peltier device & $6.28 \mathrm{rad} / \mathrm{s}$ \\
$a$ & Scaling gain for system 2 & 6 \\
$b$ & Scaling gain for system 3 & 6 \\
$T_{\mathrm{b}}$ & Artificial buffer for the correction & $50 \mathrm{~ms}$ \\
$K_{\mathrm{pm}}^{\mathrm{wo} 1}$ & P gain in axis not used for teleoperation (master 1) & 900.0 \\
$K_{\mathrm{dm}}^{\mathrm{wo} 1}$ & D gain in axis not used for teleoperation (master 1) & 100.0 \\
$K_{\mathrm{pm}}^{\mathrm{wo}}$ & P gain in axis not used for teleoperation (master 2 \& slave) & 1600.0 \\
$K_{\mathrm{dm}}^{\mathrm{wo}}$ & D gain in axis not used for teleoperation (master 2 \& slave) & 80.0 \\
\hline \hline
\end{tabular}

Red and green lines represent the response of master systems, while blue lines show the response of the slave system. The effect of the modes not decoupled can especially be seen in the position and temperature responses. It takes several seconds for each response to converging to the same response, especially, the temperature response takes about 10 seconds for the convergence. The reason is the controllers in the conventional method is interfering with each other. The effect of external force appears inside the position control loop, and the external force affects the response of temperature control.

On the contrary, the experimental results of the proposed method are shown in Figs. 18 and 19. Compared to the conventional method, position and temperature errors between subsystems are well suppressed. This is because the modes are decoupled. As for the temperature response, the convergence time was shortened up to 3 seconds which is three times faster compared to the conventional method. The thermal response of master 1 is oscillatory because of the noise caused by the power supply. It can also be seen that the timing of presenting each sensation was collected by inserting the buffer to the modal transformation matrix of force sensation. The operator was able to feel the hardness and the coldness of the contacting object more naturally in the proposed 

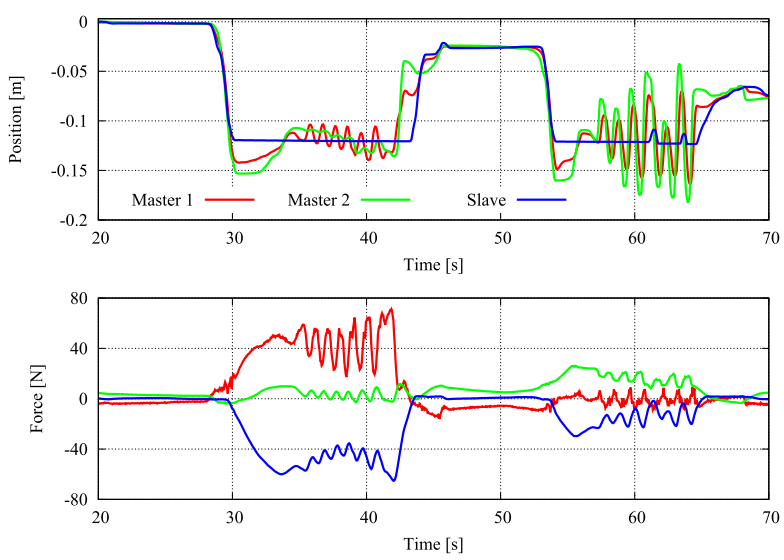

Fig. 16. Experimental results of transmitting tactile sensation using conventional $4 \mathrm{ch}$ method
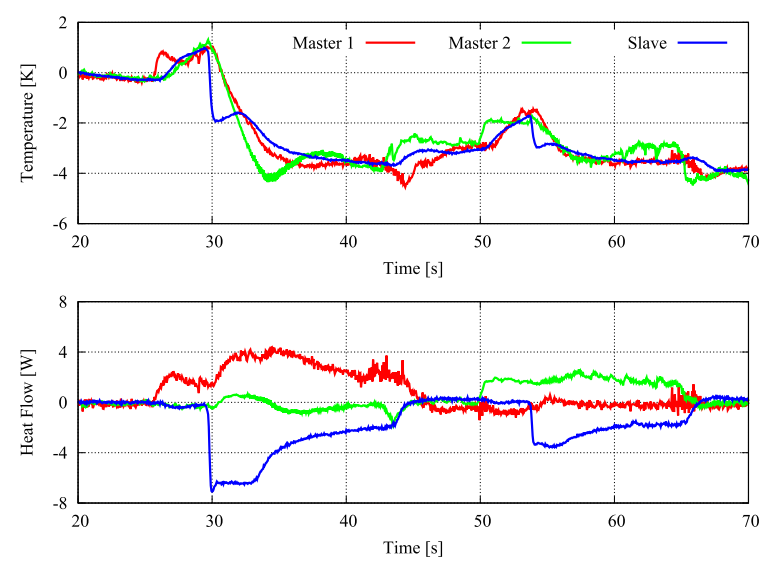

Fig. 17. Experimental results of transmitting thermal sensation using conventional $4 \mathrm{ch}$ method

method. Therefore, it can be said that precise multimodal sensation transmission was realized in the proposed method. However, there are still some errors in the transient responses. This is mainly because of the modeling errors of systems. The temperature errors in the transient response are mainly due to the low cut-off frequency of the disturbance observer for the Peltier device.

\section{Conclusions}

In this paper, the method of simultaneously presenting thermal and tactile sensations to multiple remote places using multilateral control was proposed. It became able to treat the robot manipulator and the Peltier device in the same manner by implementing the disturbance observer. By using the proposed matrix, the effect of interference because of time delay was eliminated and motion scaling was also realized to compensate the difference of motion range of each manipulator. The paper revealed that the proposed method is effective to both tactile and thermal sensation transmission. In order to consider the motion range of a robot manipulator, the scaling gain was inserted to the modal transformation matrix. Tactile sensation information was artificially buffered to correct the difference of the response speed between two actuators. The buffering time of tactile sensation for collecting the timing of the presentation should be changed according to the temperature difference of an operator and a contact object.
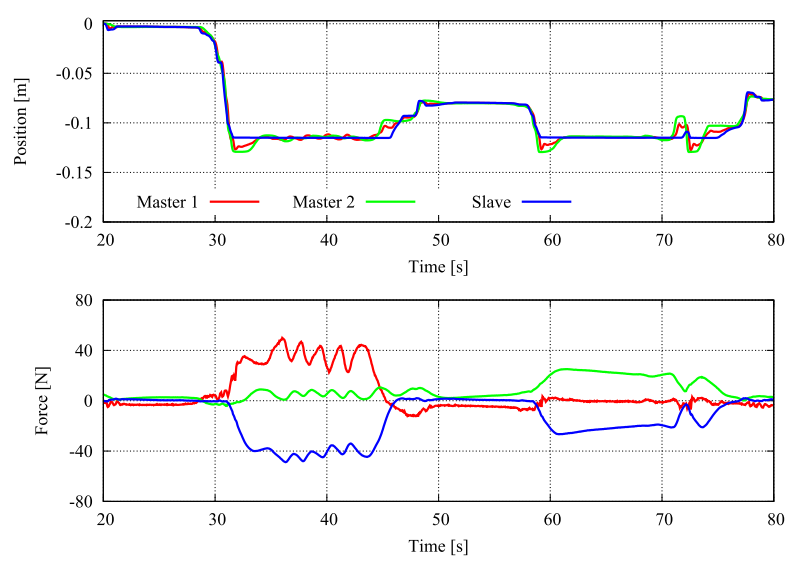

Fig. 18. Experimental results of transmitting tactile sensation using the proposed method
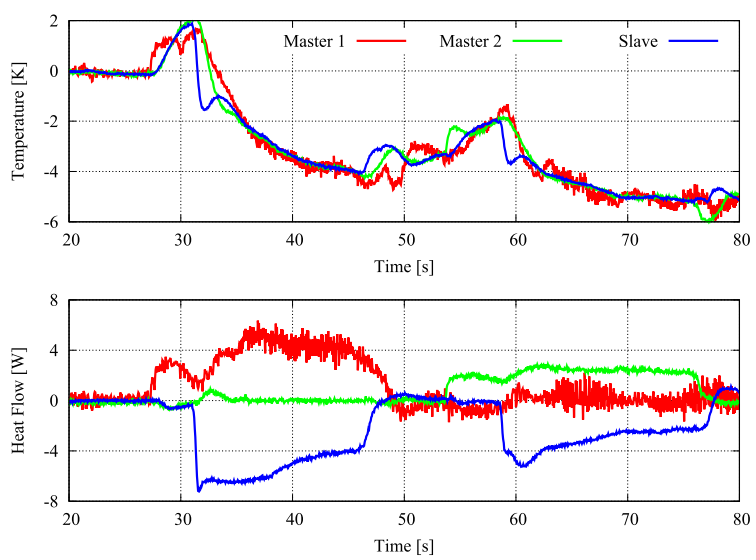

Fig. 19. Experimental results of transmitting thermal sensation using the proposed method

One of the future works includes enlarging the cut-off frequency of the heat disturbance observer. The cut-off frequency is low due to the sensor noise and the modeling error. The effect of the Joule heat will be taken into account in the modeling. As for the sensor noise, a filter other than a low-pass filter such as a Kalman filter will be implemented to suppress the effect. The construction of a multilateral teleoperation system whose operation is independent of the other master systems will be another future work. Considering the application, there are situations where the operators try to operate each system in different directions.

\section{Acknowledgment}

This work was partially supported by JSPS KAKENHI Grant Number $18 \mathrm{H} 03784$.

\section{References}

( 1 ) P.F. Hokayem and M.W. Spong: "Bilateral teleoperation: An historical survey", Automatica, Vol.42, No.12, pp.2035-2057 (2006)

( 2 ) R.C. Goertz: "Mechanical master-slave manipulator", Nucleonics, Vol.12, No.11, pp.45-46 (1954)

( 3 ) L. Eusebi and C. Melchiorri: "Force reflecting telemanipulators with timedelay: Stability analysis and control design", IEEE Trans. Robot. Autom., Vol.14, No.4, pp.635-640 (1998)

( 4 ) F. Mobasser, K. Hashtrudi-Zaad, and S. Salcudean: "Impedance reflecting rate mode teleoperation", in Proc. IEEE ICRA, Vol.3, pp.3296-3302 (2003)

( 5 ) S. Katsura, W. Iida, and K. Ohnishi: "Medical mechatronics: An application to haptic forceps", Annu. Rev. in Control, Vol.29, No.2, pp.237-245 (2005) 
( 6 ) K. Ohishi, K. Ohnishi, and K. Miyachi: "Torque-speed regulation of DC motor based on load torque estimation method", in Proc. IEEJ IPEC, pp.12091218 (1983)

( 7 ) S. Katsura, Y. Matsumoto, and K. Ohnishi: "Realization of "law of action and reaction" by multilateral control", IEEE Trans. Ind. Electron., Vol.52, No.5, pp.1196-1205 (2005)

( 8 ) B. Khademian and K. Hashtrudi-Zaad: "Dual-user teleoperation systems: New multilateral shared control architecture and kinesthetic performance measures", IEEE/ASME Trans. Mechatronics, Vol.17, No.5, pp.895-906 (2012)

(9) S. Katsura and K. Ohnishi: "A realization of haptic training system by multilateral control”, IEEE Trans. Ind. Electron., Vol.53, No.6, pp.1935-1942 (2006)

(10) S. Katsura and K. Ohnishi: "Quarry of modal information from environment for advanced motion control", IEEJ J. Ind. Appl., Vol.126, No.4, pp.372-378 (2006)

(11) H. Morimitsu and S. Katsura: "Construction of thermal multilateral control system based on quarry matirx", in Proc. IEEJ Ann. Mtg., No.4, pp.386-387 (2013)

(12) K. Natori, S. Katsura, T. Tsuji, and K. Ohnishi: "Analysis of bilateral systems with time delay", in Proc. IEEE ISIE, Vol.4, pp.1511-1516 (2005)

(13) J. Kim, P.H. Chang, and H.-S. Park: "Two-channel transparency-optimized control architectures in bilateral teleoperation with time delay", IEEE Trans. Control Syst. Technol., Vol.21, No.1, pp.40-51 (2013)

(14) U. Tumerdem and M. Demir: "L2 stable transparency optimized two channel teleoperation under time delay", in Proc. IEEE IECON, pp.1313-1320 (2015)

(15) H.V. Quang and J.-H. Ryu: "Stable multilateral teleoperation with time domain passivity approach", in Proc. IEEE/RSJ IROS, pp.5890-5895 (2013)

(16) D. Sun, F. Naghdy, and H. Du: "Stability control of force-reflected nonlinear multilateral teleoperation system under time-varying delays", J. Sensors, pp.1-17 (2016)

(17) T. Kanno and Y. Yokokohji: "Multilateral teleoperation control over timedelayed computer networks using wave variables", in Proc. IEEE HAPTICS, pp.125-131 (2012)

(18) A. Yamaguchi and K. Ohnishi: "Improvement of operationality for multilateral control system under constant time delay", in Proc. IEEE AMC, pp.538543 (2008)

(19) S. Nishimura and S. Katsura: "Multilateral control under time delay for decoupling force and velocity controllers", in Proc. IEEE ISIE, pp.1258-1263 (2016)

(20) H. Morimitsu and S. Katsura: "Control of thermal conductance of peltier device using heat disturbance observer", IEEJ J. Ind. Appl., Vol.132, No.3, pp.333-339 (2012)

(21) T. Murakami and K. Ohnishi: "A study of stability and workspace decoupling control based on robust control in multi-degrees-of-freedom robot", IEEJ J. Ind. Appl., Vol.113, No.5, pp.639-646 (1993)

Satoshi Nishimura (Member) received his B.E. degree in system de-

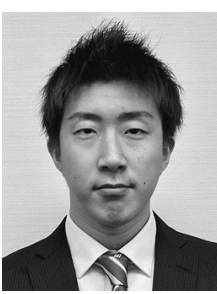
sign engineering and the M.E. and Ph.D. degrees in integrated design engineering from Keio University, Yokohama, Japan, in 2013, 2014, and 2017, respectively. From 2016 to 2018, he was a Research Fellow of the Japan Society for the Promotion of Science (JSPS). His research interests include real-world haptics, motion control, and multi-systems. He is a Member of IEEJ, as well as IEEE.

Yukiko Osawa

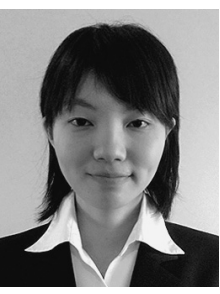

Hiroki Kurumatani (Student Member) received his B.E. degree in

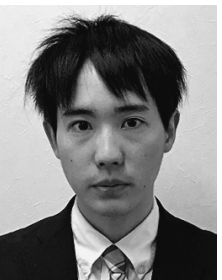
system design engineering and the M.E. degree in integrated design engineering from Keio University, Yokohama, Japan, in 2015 and 2017, respectively. Since 2017, he has been a Ph.D. course student at Keio University, Yokohama, Japan. His research interests include motion control, power electronics, heterogeneous computing and system design. He is a Student Member of IEEJ, as well as IEEE.

Yuki Nagatsu (Member) received the B.E. degree in system design

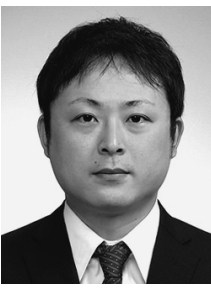
engineering and the M.E. and Ph.D. degrees in integrated design engineering from Keio University, Yokohama, Japan, in 2012, 2014 and 2017, respectively. He is currently with the Department of Electrical, Electronic, and Communication Engineering, Chuo University, Tokyo, Japan. His research interests include motion control, robotics, real-world haptics, intelligent space and human support systems. He is a Member of The Institute of Electrical Engineers of Japan (IEEJ) and Robotics Society of Japan (RSJ).

Kazumasa Miura (Member) received the B.E. degree in system de-

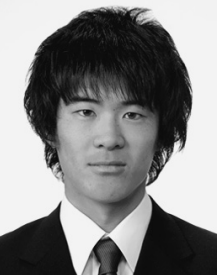
ign engineering and the M.E. and Ph.D. degrees in integrated design engineering from Keio University, Yokohama, Japan, in 2012, 2014, and 2017, respectively. He is currently with the Laboratory for Machine Tools and Production Engineering (WZL) of RWTH Aachen University, Aachen, Germany. His research interests include motion control, mechatronics, robotics, haptics, and manufacturing engineering.

Seiichiro Katsura (Senior Member) received the B.E. degree in sys-

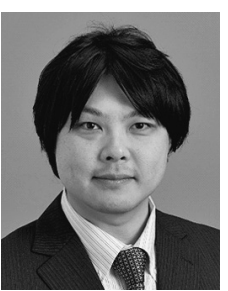
em design engineering and the M.E. and Ph.D. degrees in integrated design engineering from Keio University, Yokohama, Japan, in 2001, 2002 and 2004, respectively. From 2003 to 2005, he was a Research Fellow of the Japan Society for the Promotion of Science (JSPS). From 2005 to 2008, he worked at Nagaoka University of Technology, Nagaoka, Niigata, Japan. Since 2008, he has been at Keio University, Yokohama, Japan. In 2017, he was a Visiting Researcher with the Laboratory for Machine Tools and Production Engineering (WZL) of RWTH Aachen University, Aachen, Germany. His research interests include applied abstraction, human support, wave system, systems energy conversion, and electromechanical integration systems. Prof. Katsura serves as an Associate Editor of the IEEE Transactions on Industrial Electronics. He was the recipient of the IEEJ Distinguished Paper Awards in 2003 and 2017, IEEE Industrial Electronics Society Best Conference Paper Award in 2012, and JSPS Prize in 2016 